\title{
Community based occupational therapy for patients with dementia and their care givers: randomised controlled trial
}

\author{
Maud J L Graff, Myrra J M Vernooij-Dassen, Marjolein Thijssen, Joost Dekker, \\ Willibrord H L Hoefnagels, Marcel G M Olde Rikkert
}

Editorial by Lawlor and Golden

Research Group for Allied Health Care, Department of Allied Health Care Disciplines,

Occupational

Therapy, University

Medical Center

Nijmegen, PO Box

$9101,6500 \mathrm{HB}$

Nijmegen,

Netherlands

Maud J L Graff

scientific researcher in

allied health care

research

Marjolein Thijssen research assistant

Center for Quality of Care Research/

Alzheimer Center

Nijmegen,

University Medical

Center Nijmegen,

PO Box 9101, 6525

JV Nijmegen

Myrra J M

Vernooij-Dassen

senior researcher in

senior researcher in

research

Department of

Rehabilitation

Medicine, EMGO

Institute, $\mathrm{VU}$

University Medical

Center Amsterdam,

PO Box 7057, 1007

MB Amsterdam

Joost Dekker

professor in aU

Department of

Geriatrics/Alzheimer

Center Nijmegen,

931 University

Medical Cente

Nijmegen, PO Box

$9101,6500 \mathrm{HB}$

Nijmegen

Willibrord H I

Hoefnagels

professor in geriatrics

Marcel G M Olde

Rikkert

professor in geriatrics

Correspondence to:

M J L Graff

m.graff@ergo.umcn.nl

BMJ 2006;333:1196-9

\section{Abstract}

Objective To determine the effectiveness of community based occupational therapy on daily functioning of patients with dementia and the sense of competence of their care givers.

Design Single blind randomised controlled trial. Assessors were blinded for treatment allocation. Setting Memory clinic and day clinic of a geriatrics department and participants' homes.

Participants 135 patients aged $\geq 65$ with mild to moderate dementia living in the community and their primary care givers.

Interventions 10 sessions of occupational therapy over five weeks, including cognitive and behavioural interventions, to train patients in the use of aids to compensate for cognitive decline and care givers in coping behaviours and supervision.

Main outcome measures Patients' daily functioning assessed with the assessment of motor and process skills (AMPS) and interview of deterioration in daily activities in dementia (IDDD). Care giver burden assessed with the sense of competence questionnaire (SCQ). Participants were evaluated at baseline, six weeks, and three months.

Results Scores improved significantly relative to baseline in patients and care givers in the intervention group compared with the controls (differences were 1.5 (95\% confidence interval 1.3 to 1.7$)$ for the process scale; -11.7 ( -13.6 to -9.7$)$ for the performance scale; and (11.0; 9.2 to 12.8) for the competence scale). This improvement was still significant at three months. The number needed to treat to reach a clinically relevant improvement in motor and process skills score was 1.3 (1.2 to 1.4) at six weeks. Effect sizes were 2.5, 2.3, and 1.2, respectively, at six weeks and 2.7, 2.4, and 0.8 , respectively, at 12 weeks.

Conclusions Occupational therapy improved patients' daily functioning and reduced the burden on the care giver, despite the patients' limited learning ability. Effects were still present at 12 weeks, which justifies implementation of this intervention. Trial registration Clinical Trials NCT00295152.

\section{Introduction}

Dementia has far reaching consequences for patients and their primary care givers and is currently a major driver of costs in health care and social systems in developed countries. ${ }^{1}$ Care givers often experience feelings of helplessness, social isolation, and loss of autonomy. ${ }^{2-4}$ Unfortunately, drugs are not yet effective in improving the symptoms of dementia, and non-pharmacological strategies are generally more time consuming and not widely available. A systematic review found non-pharmacological interventions to produce effect sizes in behaviour similar or larger to those seen with cholinesterase inhibitors, the currently available drug treatment, but without any side effects. ${ }^{5}$ Occupational therapy is also said to be effective in dementia. ${ }^{6-9}$ The primary focus of such a therapy is to improve patients' ability to perform activities of daily living and hence promote independence and participation in social activities ${ }^{4}{ }^{9}$ and to reduce the burden on the care giver by increasing their sense of competence and ability to handle the behavioural problems they encounter. ${ }^{4-10}$ These outcomes are increasingly being considered equally or even more clinically relevant than measures of cognitive outcome. ${ }^{11}$

We conducted a randomised controlled trial to study the effects of community based occupational therapy on the daily functioning of patients with dementia and on the sense of competence among their primary care givers.

\section{Methods}

\section{Participants}

From April 2001 to January 2005, we recruited 135 people from the memory clinic and the day clinic of a department of geriatrics. Patients were included if they were aged $\geq 65$, had been diagnosed with mild to moderate dementia, were living in the community, and had a primary care giver who cared for them at least once a week. We excluded patients with a score $>12$ on the geriatric depression scale, ${ }^{12}$ severe behavioural or psychological symptoms in dementia (BPSD), and severe illnesses as judged by a geriatrician and those in whom occupational therapy goals could not be defined or who were not on stable treatment of a dementia drug (that is, less than three months on the same dose of a cholinesterase inhibitor or memantine). We also excluded care givers with severe illnesses.

The geriatrician gave all eligible patients and primary care givers written and verbal information, and the researcher explained the assessment instruments and gave examples. After being given the time needed to make a decision and if they wanted to take part, the patient and care giver signed the informed consent form in a second meeting with the researcher.

\section{Randomisation and procedures}

Patients were randomly assigned by blocked randomisation (block size 4) to the intervention (10 sessions of

Details of the research protocol can be found on bmi.com

This is the abridged version of an article that was posted on 10.1136/bmj.39001.688843.BE 
occupational therapy at home over five weeks) or control group (no occupational therapy), which was stratified by level of dementia (mild or moderate). In this single blind randomised controlled trial, patients and care givers were aware of the treatment assigned. The assessors (MT or MJLG) were blinded to group allocation. Patients and care givers were asked before each assessment not to inform the assessors about the intervention. To check the success or failure of the blinding after each measurement the assessors were asked if they had been told or knew for sure to which group each patient had been allocated. The total study period per patient was 12 weeks from the moment of inclusion. The control group received occupational therapy after completion of the study (12 weeks later).

\section{Intervention}

The study intervention was developed in a consensus process and was implemented by experienced occupational therapists who had been trained (for about 80 hours) and were experienced (for at least 240 hours) in delivering treatment according to a client centred occupational therapy guideline for patients with dementia. ${ }^{913}$ Treatment consisted of 10 one hour sessions held over five weeks and focused on both patients and their primary care givers. The total time spent for the intervention, including the time spent for treatment at home (10 hours), narrative analysis, reports, and multidisciplinary briefing (details on bmj. com), was about 18 hours per patient and care giver together. $^{4}$

\section{Outcome assessments and measures}

We assessed patients and their primary care givers at baseline before the intervention and six weeks (effect measurement) and 12 weeks (follow-up measurement) later. Our primary outcome measure for patients was daily functioning assessed with the process scale of the assessment of motor and process skills,${ }^{14}$ in which scores range from -3 to 4 (higher scores indicate better process skills), and with the performance scale of the interview of deterioration of daily activities in dementia, ${ }^{15}$ in which scores range from 0 to 44 (lower scores indicate less need for assistance). The outcome for primary care givers was sense of competence assessed with the sense of competence questionnaire, ${ }^{16}$ in which scores ranged from 27 to 135 (higher scores denote greater sense of competence).

We collected information on the age, sex, and educational level of the patient and care giver at baseline. In patients we assessed co-morbidity (cumulative illness rating scale for geriatrics ${ }^{17}$ ), depressive mood (geriatric depression scale ${ }^{12}$ ), cognition (mini-mental state examination ${ }^{18}$ ), and behaviour (revised memory and behavioural problems checklist $\left.{ }^{15}{ }^{19}\right)$. We also assessed the relationship between care givers and patients and depression in care givers (Center for Epidemiologic Studies depression scale $\left.{ }^{20}\right)$.

\section{Statistical analysis}

We used analyses of covariance of the primary outcome measures (process scale, performance score, and competence at six weeks) to determine the main effects based on an intention to treat analysis of all available data, applying the last observation carried forward method for dropouts. Treatment differences between baseline and six weeks were computed by
Baseline characteristics of patients and care givers

\begin{tabular}{lcc} 
& $\begin{array}{c}\text { Occupational } \\
\text { therapy }(\mathbf{n = 6 8})\end{array}$ & Control $(\mathbf{n = 6 7 )}$ \\
\hline Mean (SD) age (years): & $79.1(6.2)$ & $77.1(6.3)$ \\
\hline Patient & $66.0(15.3)$ & $61.3(15.4)$ \\
\hline Primary care giver & & \\
\hline Sex (M/F): & $29 / 39$ & $31 / 36$ \\
\hline Patient & $22 / 46$ & $18 / 49$ \\
\hline Primary care giver & & \\
\hline Relation of care giver to patient: & 41 & 38 \\
\hline Partner & 22 & 21 \\
\hline Daughter & 5 & 8 \\
\hline Other & $19.0(5.7)$ & $19.0(4.0)$ \\
\hline Mean (SD) scores on assessment scales: & $11.6(4.3)$ \\
\hline Mini-mental state & $10.7(3.5)$ & $7.5(3.0)$ \\
\hline CIRS-G & $6.9(3.0)$ & $5.0(6.0)$ \\
\hline Geriatric depression scale & $5.6(5.3)$ & $1.1(1.0)$ \\
\hline RMBPC frequency & $1.0(1.1)$ & $0.3(0.8)$ \\
\hline AMPS-motor & $0.2(0.8)$ & $24.5(8.7)$ \\
\hline AMPS-process & $23.5(7.9)$ & $8.1(4.6)$ \\
\hline IDDD-performance & $8.3(6.2)$ & $27.1(4.2)$ \\
\hline Cornell depression scale & $27.3(5.1)$ & $11.4(7.2)$ \\
\hline Brief cognitive rating scale & $89.7(14.9)$ & $(13.6)$ \\
\hline Sense of competence & $11.7(8.3)$ & \\
\hline CES-D & & \\
\hline
\end{tabular}

CIRS-G=cumulative illness rating scale for geriatrics; RMBPC=revised memory and behavioural problems checklist; AMPS=assessment of motor and process skills (higher scores indicate better skills); IDDD=interview of deterioration in daily activities in dementia (lower scores indicate less need for help): BCRS=brief cognitive rating scale; CES-D=Center for Epidemiologic Studies depression scale.

analysis of covariance, with age, sex, relation to patient, other care givers, and baseline scores on the comorbidity, depression, cognition, and behaviour scales and the outcome variable as covariates. We carried out secondary analyses on the primary outcome measures at 12 weeks (conditional analysis: only in case of positive effects at six weeks).

The study was powered to detect a clinically relevant difference in change over time of 0.5 points on the process scale between the two groups, 20\% improvement on the performance interview, and a 5 point difference on the competence scale, with a power of $80 \%$ on the basis of one sided testing, a standard deviation of 0.8 on the process scale, and $n \geq 100$. The power calculation was based on earlier data ${ }^{9}$ and on the minimal clinically relevant differences in the primary outcomes as defined in the measurement guideline for the process scale, which describes 0.5 points as clinically relevant, ${ }^{14}$ and the measurement guideline for the performance interview. ${ }^{15}$

\section{Results}

We evaluated 275 consecutive patients diagnosed with dementia and living in the community for eligibility. The baseline characteristics of patients and care givers were well matched between the two groups. We corrected for age differences (mean ages were lower by 2.0 (patients) and 4.7 (care givers) years in the control group) in the analysis of covariance (table).

\section{Outcomes at six weeks}

There were significant differences between the groups on all primary outcome variables at six weeks. Patients who received occupational therapy functioned significantly better in daily life than those who did not (for 
intervention $v$ control, mean process scores were 1.2 (SD 0.7) $v 0.2$ (SD 0.8), and the mean performance interview scores were 14.4 (SD 6.1) v 25.3 (SD 8.6), figure). The difference between the groups was significant $(1.5,95 \%$ confidence interval 1.3 to 1.7 , for the process scale; $-11.7,-13.6$ to -9.7 , for the performance interview; see bmj.com). Primary care givers who received occupational therapy felt significantly more competent than those who did not (mean competence score 104.6 (SD 13.4) v 88.4 (SD 13.7), figure). The difference in competence scores was significant (11.0, 9.2 to 12.8 ).

Overall, $84 \%$ in the intervention group and 9\% in the control group achieved a clinically relevant improvement on the process outcome, the figures being $78 \% v 12 \%$ for the performance interview. For the care givers $58 \%$ and $18 \%$ had a clinically relevant improvement in feelings of competence. For all three outcomes together $47 \%$ in the intervention group and $2 \%$ in the control group achieved a clinically relevant difference. The number needed to treat was 1.3 (1.2 to 1.4) for the process outcome, 1.5 (1.4 to 1.6) for the performance outcome, and 2.5 (2.3 to 2.7) for competence outcome (see bmj.com). The number needed to treat for all three primary outcomes together was 2.2 (2.1 to 2.3). The effect sizes at six weeks were 2.5, 2.3, and 1.2, respectively (see bmj.com). The per protocol analyses at six weeks showed effect sizes of 3.2, 2.3, and 1.2 , respectively. In $82 \%$ of the cases blinding was successful, and in $18 \%(n=21)$ the assessors knew the treatment allocation.

\section{Outcomes at 12 weeks}

Differences between the groups on all outcome variables were also evident at 12 weeks (details are on bmj.com).

\section{Discussion}

In this randomised controlled trial we found evidence that 10 sessions of community occupational therapy, given over five weeks, improves the daily functioning of patients with dementia and diminishes the burden of care on their primary care givers. The process skills and need for assistance in performing daily activities improved in patients, and their care givers felt more competent at six weeks, and these beneficial effects remained so at 12 weeks. A similar positive effect of occupational therapy was reported earlier in stroke patients. ${ }^{21}$ The improvement was also clinically relevant, meeting predefined criteria for clinical relevance and highly effective with low numbers needed to treat. At six weeks, the process outcome score of patients was higher than that associated with independent functioning (cut-off score of 1.0) and remained so at 12 weeks. Moreover, the effect sizes of all primary outcomes were higher than those found in trials of drugs or other psychosocial interventions for people with dementia. ${ }^{5}$ We believe that the benefit was sustained because a component of the intervention was to train care givers in providing the supervision patients needed to sustain their performance of daily activities. The intervention also provided individualised support to care givers, which earlier studies have also shown to be effective. ${ }^{22-24}$
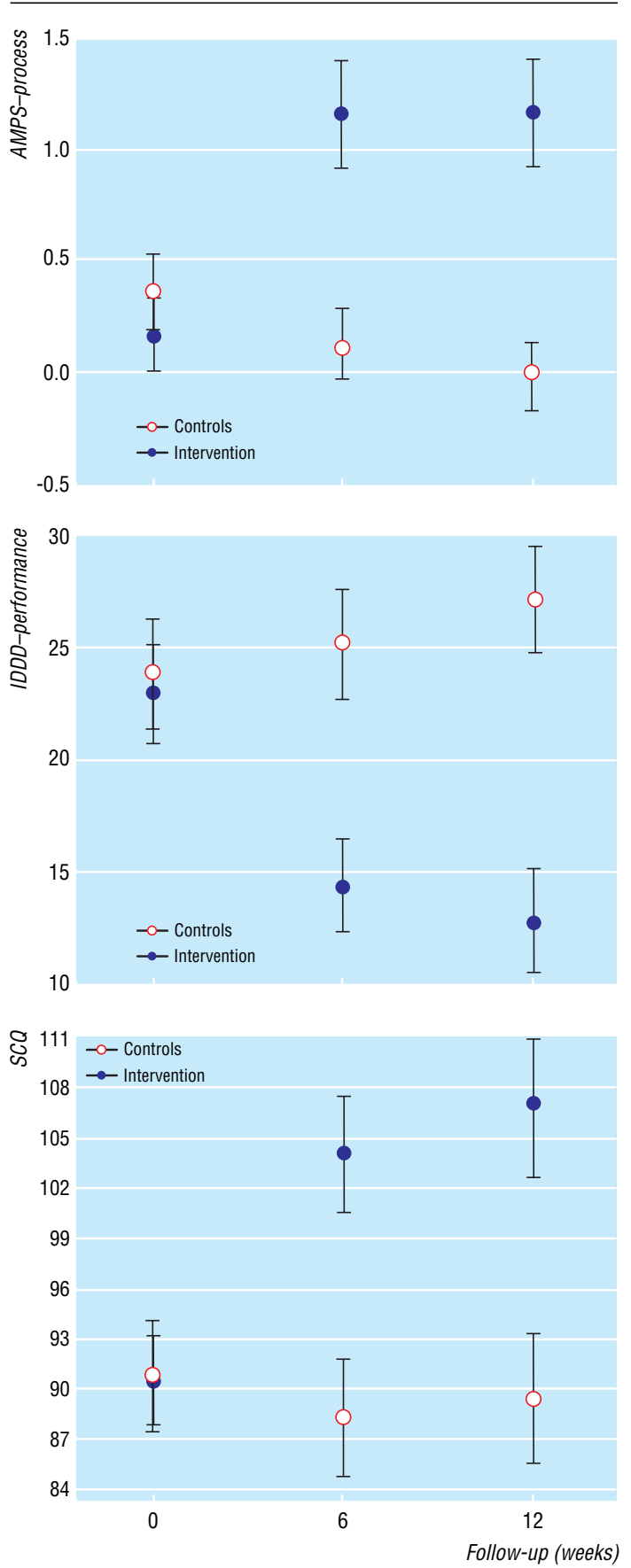

Mean (95\% confidence interval) scores on assessment of motor and process skills (AMPS), performance interview (IDDD), and sense of competence (SCQ) in care givers at baseline, six, and 12 weeks in intervention and control groups

\section{Strengths and weaknesses}

Two earlier studies evaluated occupational therapy in patients with dementia ${ }^{67}$ but their methodological quality was poor. ${ }^{25}$ A recent study by Gitlin et al had similar results on care giver outcome after a community occupational therapy programme for patients with dementia and their primary care givers. ${ }^{8}$ The outcomes of our study were also expressed in effect sizes as recommended by Luijpen et al, ${ }^{5}$ which enables comparison with drug and non-drug interventions. Our design was based on a pilot study of the 


\section{What is already known on this topic}

Effective treatment for patients with dementia and their care givers should lead to improvement in activities of daily living and diminished burden on the care giver

Drugs are not effective in improving the symptoms of dementia and non-pharmacological strategies have similar effect sizes and no side effects but are generally more time consuming

\section{What this study adds}

Ten sessions of community occupational therapy over five weeks improved the daily functioning of patients with dementia, despite their limited learning abilities, and reduced the burden on their informal care givers

The effect sizes of all primary outcomes were higher than those found in trials of drugs or other psychosocial interventions, and these effects were still present at three months

intervention protocol. ${ }^{9}$ The occupational therapy intervention was based on a guideline developed on the basis of consensus among a national panel of qualified and experienced occupational therapists. ${ }^{43}$ We had a high follow-up rate at 12 weeks, possibly because our study was directly relevant to the daily lives of patients and their care givers. A limitation of our study design is that, as with some other types of treatment, we could not carry out a double blind study because the patients and their care givers knew which therapy they received, nor was it possible to blind occupational therapists to treatments. We tried to maintain masked conditions for assessment, however, which succeeded for $80 \%$ of the cases. ${ }^{26}$ For this reason, we believe that our results are not greatly affected by observer bias. Another potential limitation is that our sample might not be representative of all patients with mild to moderate dementia in our health region as participants were recruited primarily from the outpatient clinics of the university hospital and not from other institutions or directly from general practices. We chose this recruitment strategy because we wanted to achieve uniformity in terms of screening and diagnosis to facilitate comparison with other national and international studies. The size of the effects is promising for implementation in other settings as well.

Because outcomes such as improvement in activities of daily living and sense of competence are associated with a decrease in need for assistance, ${ }^{15}$ we believe that, in the long term, occupational therapy will result in less dependence on social and healthcare resources and less need for institutionalisation. ${ }^{23}$ The training in effective use of the intervention (at least 80 hours) and the intervention itself is quite comprehensive (time spent for treatment at home, narrative analysis, reports, and multidisciplinary briefing is about 18 hours per patient and care giver). We believe, however, that it is worth implementing in clinical practice because of its relevant effects and high efficacy, which makes it reasonable to expect cost effectiveness in clinical practice.

We thank all participants for their contribution and Jana Zajec and Patricia Verstraten for all occupational therapy treatments. Contributors: See bmj.com.

Funding: Dutch Alzheimer Association with financial support of the Radboud University Nijmegen Medical Center and the Dutch Occupational Therapy Association.

Competing interests: None declared.

Ethical approval: Medical ethics committee of the UMCN of Nijmegen and Arnhem, number CWOM0012-0292.

1 Wimo A, Jonsson L, Winblad B. An estimate of the worldwide prevalence and direct costs of dementia in 2003. Dement Geriatr Cogn Disord 2006;21:175-81.

2 Coen J.Dementia and caregiving. J Health Gain 1998:2:5-6.

3 Jepson C, McCorkle R, Adler D, Nuamah I, Lusk E. Effects of home care on caregivers' psychosocial status. Image J Nurs Sch 1999;31:115-20.

4 Graff MJL, Vernooij-Dassen MJFJ, Zajec J, Olde Rikkert MGM, Hoefnagels WHL, Dekker J. How can occupational therapy improve the daily performance and communication of an older patient with dementia and his primary caregiver? A case study. Dementia 2006;5:503-32.

5 Luijpen MW, Scherder EJ, Van Someren EJ, Swaab DF, Sergeant JA. Nonpharmacological interventions in cognitively impaired and demented patients - a comparison with cholinesterase inhibitors. Rev Neurosci 2003;14:343-68.

6 Burgener SC, Bakas T, Murray C, Dunahee J, Tossey S. Effective caregiving approaches for patients with Alzheimer's disease. Geriatr Nurs 1998; 19:121-52.

7 Gitlin LN, Corcoran M, Winter L, Boyce A, Hauck WW. A randomized, controlled trial of a home environment intervention: effect on efficacy and upset in caregivers and on daily functioning of persons with dementia. Gerontologist 2001;41:4-14.

8 Gitlin LN, Hauck WW, Dennis MP, Winter L. Maintenance of effects of the home environmental skill-building program for family caregivers and individuals with Alzheimer's disease and related disorders. J Gerontol 2005;60A:368-74.

9 Graff MJL, Vernooij-Dassen MJFJ, Hoefnagels WHL, Dekker J, Witte de LP. Occupational therapy at home for older individuals with mild to LP. Occupational therapy at home for older individuals with mild to
moderate cognitive impairments and their primary caregivers: a pilot moderate cognitive impairments and
study. Occup Ther J Res 2003;23:155-63.

10 Corcoran M, Gitlin L. Dementia management: an occupational therapy home-based intervention for caregivers. Am J Occup Ther 1992;46:801-8. 11 Rockwood K, Gauthier S. Trial designs and outcomes in dementia therapeutic research. London: Taylor and Francis, 2006.

12 Brink TL, Yesavage JA, Lum O, Heersema P, Adey MB, Rose TL. Screening tests for geriatric depression. Clin Gerontol 1982;1:37-43.

13 Graff MJL, Melick van MBM. The development, testing and implementation of an occupational therapy guideline. The guideline for the OT diagnosis and treatment of older persons with cognitive impairments. Ned Tijdschr Ergother 2000;28:169-74. [In Dutch]

14 Fisher AG. Assessment of motor and process skills. Fort Collins, CO: Three Stars Press, 2003.
Tessic

15 Teunisse S. Clinimetrics in dementia. Enschede, Netherlands: PrintPartners Teunisse S. Clinimat.

16 Vernooij-Dassen MJM, Persoon JM, Felling AJ. Predictors of sense of competence in caregivers of demented individuals. Soc Sci Med 1996;43:41-9.

17 Miller MD, Paradis CF. Rating chronic medical illness in burden in psychogeriatric practice and research: the cumulative illness rating scale in a geriatric residential population (CIRS-G). Psychiatr Res 1991;41:23748.

18 Folstein MF, Folstein SE, McHugh PR. Mini-mental state: a practical method for grading the cognitive state of patients for the clinician.J Psymethod for grading the co
chiatr Res 1975;12:189-98.

19 Teri L, Truax P, Logsdon R, Uomoto J, Zarit S, Vitaliano PP. Assessment Teri L, Truax P, Logsdon R, Uomoto J, Zarit S, Vitaliano PP. Assessment
of behavioral problems in dementia: the revised memory and behavior problems checklist. Psychol Aging 1992;7:622-31.

20 Radloff LS. The CES-D scale: a self-report depression scale for research in the general population. Applied Psychol Measurement 1997;1:385-401.

21 Walker MF, Gladman JRF, Lincoln NB, Siemonsma P, Whiteley T. Occupational therapy for stroke patients not admitted to hospital: a randomised controlled trial. Lancet 1999;354:278-80.

22 Witlach CJ, Zarit SH, Von Eye A. Efficacy of interventions with caregivers. A reanalysis. Gerontologists 1991;31:9-14.

23 Vernooij-Dassen M, Lamers C, Bor J, Felling A, Grol R. Prognostic factors of effectiveness of a support program for caregivers of dementia patients. Int J Aging Hum Dev 2000;51:259-74.

24 Fossey J, Ballard C, Juszczak E, James I, Alder N, Jacoby R, et al. Effect of enhanced psychosocial care on antipsychotic use in nursing home residents with severe dementia: cluster randomised trial. BMJ 2006;332:756-61.

25 Steultjens EMJ, Dekker J, Bouter L, Jellema S, Bakker, EB, vandenEnde CHM. Occupational therapy for community dwelling elderly people: a systematic review. Age Ageing 2004;33:453-60.

26 Siemonsma PC, Walker MF. Practical guidelines for independent assessment in randomised controlled trials (RCTs) of rehabilitation. Clin Rehabil 1997;11:273-9.

(Accepted 6 October 2006)

doi 10.1136/bmj.39001.688843.BE 